\title{
Enhancing Efficiency of Perovskite Solar Cells via N-doped Graphene: Crystal Modification and Surface Passivation
}

\author{
Mahboubeh Hadadian, Juan-Pablo Correa-Baena, * Elaheh K. Goharshadi,* \\ Amita Ummadisingu, Ji-Youn Seo, Jingshan Luo, Somayeh Gholipour, Shaik M. Zakeeruddin, \\ Michael Saliba, Antonio Abate, Michael Grätzel, and Anders Hagfeldt**
}

Thin-film photovoltaics (PVs) are some of the most cost-effective approaches to convert sunlight directly into electric power. Over the past few years, perovskite solar cells (PSCs) of general formula $\mathrm{ABX}_{3}$ (where $\mathrm{A}$ is an organic/inorganic cation, $\mathrm{B}$ is a metal cation, and $\mathrm{X}$ is a halide anion), have emerged at the forefront of thin-film PVs due to their high power conversion efficiency and low-cost fabrication. ${ }^{[1,2]}$ Since the pioneering work of Miyasaka and co-workers in 2009, ${ }^{[3]}$ PSCs have witnessed impressive advances with the solar-to-electrical power conversion efficiency (PCE) improving from 3.8\% to a certified value of $22.1 \%$ on laboratory-scale cells in 2016. ${ }^{[4]}$ Such a high value in PCE can be attributed to the unique properties of the material, including tunable optical band gap, long charge diffusion length, low exciton binding energy, and low charge recombination rate. ${ }^{[5,6]}$ The dominant architecture of the PSC consists of a perovskite light-absorbing layer sandwiched between a hole-transporting material (HTM) such as Spiro-OMeTAD

M. Hadadian, Dr. J.-P. Correa-Baena,

S. Gholipour, Prof. A. Hagfeldt

Laboratory of Photomolecular Science

Institute of Chemical Sciences and Engineering

École Polytechnique Fédérale de Lausanne

1015 Lausanne, Switzerland

E-mail: juan.correa@epfl.ch; anders.hagfeldt@epfl.ch

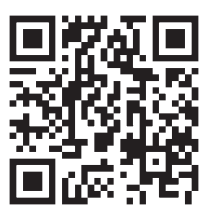

M. Hadadian, Prof. E. K. Goharshadi

Department of Chemistry

Ferdowsi University of Mashhad

Mashhad 91779, Iran

E-mail: gohari@um.ac.ir

Prof. E. K. Goharshadi

Center of Nano Research

Ferdowsi University of Mashhad

Mashhad 91779, Iran

A. Ummadisingu, J.-Y. Seo, Dr. J. Luo, Dr. S. M. Zakeeruddin,

Dr. M. Saliba, Dr. A. Abate, Prof. M. Grätzel

Laboratory of Photonics and Interfaces

Institute of Chemical Sciences and Engineering

École Polytechnique Fédérale de Lausanne

1015 Lausanne, Switzerland

S. Gholipour

Faculty of Physics and Chemistry

Alzahra University

Tehran 1993893973, Iran

Dr. A. Abate

Adolphe Merkle Institute

University of Fribourg Ch. du. Musée 3

$\mathrm{CH}-1700$ Fribourg, Switzerland $\left(2,2^{\prime}, 7,7^{\prime}\right.$-tetrakis ( $N, N$-bis ( $p$-methoxyphenyl)amino $)-9,9^{\prime}$ spirobifluorene) and an electron-transporting layer, often $\mathrm{TiO}_{2}$. Remarkably, by removing the $\mathrm{HTM}^{[7]}$ or by coating perovskite onto an electronically inert alumina mesoporous scaffold, ${ }^{[8]}$ the perovskite layer was found to function not only as a sensitizer but also as an ambipolar charge transporter. ${ }^{[9]}$

Morphology, grain size, and thickness are all key factors that contribute to high performance. ${ }^{[10-12]}$ Thick perovskite layers with large grains are more desirable in order to reach the maximum light-harvesting following the trend of making a single crystal perovskite layer more similar to silicon PVs. ${ }^{[0]}$ Several works have recently been published, highlighting the importance of controlling the perovskite crystal morphology towards larger grains in order to achieve high-efficiency PSCs. ${ }^{[11,13-15]}$ In our recent work, we showed that large crystals are necessary to achieve state of the art photovoltaic metrics and stabilized power output. ${ }^{[16]}$ In addition, Bi et al. ${ }^{[17]}$ reported that the poor perovskite film quality with small grain sizes is due to the quick reaction between lead halide and the cations during crystallization of perovskite from the blended precursor solution. Therefore, controlling the nucleation and crystallization process of the perovskite layer is an important route towards improving perovskite film morphology. On the other hand, due to the perovskite crystalline nature, the perovskite interfaces also influence the device performance. This has encouraged the study of passivation of surface charge trap states of the perovskite and corresponding grain boundaries by using different passivating agents such as thiophene and pyridine Lewis bases, ${ }^{[18]}$ the organic molecule iodopentafluorobenzene, ${ }^{[18]}$ fullerene, ${ }^{[19]}$ and even $\mathrm{PbI}_{2} \cdot{ }^{[20]}$

One way to improve the optoelectronic properties of perovskite materials is introducing graphene derivatives to the perovskite structure. For example, He et al. ${ }^{[21]}$ developed an in situ solution method for chemical decoration of $\mathrm{CH}_{3} \mathrm{NH}_{3} \mathrm{PbI}_{3}$ perovskites with reduced graphene oxides (RGO) to significantly improve the photodetector performance. Owing to its exceptional electrical, optical, and physical properties as well as its low-cost solution-phase production techniques, graphene has risen as a promising candidate for optoelectronic applications. ${ }^{[22,23]}$ Recently, RGO has been used in the mesoporous $\mathrm{TiO}_{2}{ }^{[24]}$ or as a HTM in planar PSC. ${ }^{[25]}$ Furthermore, the functionalization of graphene through the introduction of functional groups and chemical doping with $\mathrm{N}, \mathrm{B}$, or, S atoms opens wide opportunities to manipulate graphene properties. In particular, nitrogen is considered to be the most frequently used element for regulating the electronic and chemical properties of graphene. ${ }^{[26,27]}$ 
Here, we incorporate, for the first time, nitrogen-doped reduced graphene oxide (N-RGO) into mixed organic-inorganic halide perovskite. By introducing N-RGO to the perovskite layer, we observe a dual effect on the photovoltaic performance of PSCs. First, the presence of N-RGO in the perovskite film increases the perovskite grain size due to the slowing down the crystallization process, which translates to higher current and fill factor (FF). We also found that doped and undoped graphene sheets passivate the surface and retard the electron-hole recombination resulting in an improvement in the open-circuit voltage $\left(V_{\mathrm{OC}}\right)$, regardless of crystal size modification by $\mathrm{N}$-RGO. The resulting device exhibits an almost hysteresis-free PCE of $18.7 \%$ as compared to the reference cell with $17.3 \%$.

We fabricated N-RGO via an effective one-step hydrothermal reaction to simultaneous $\mathrm{N}$-doping and reduction of graphene oxide using urea. Figure S1a in the Supporting Information shows the schematic illustration of the structure and preparation of N-RGO starting from graphene oxide. The prepared N-RGO was composed of few-layer transparent graphene sheets with homogeneous distribution of $\mathrm{C}, \mathrm{N}$, and $\mathrm{O}$ on the surface of the graphene sheets (see Figure S1b-g in the Supporting Information).

Three common bonding configurations within the carbon lattice are possible when nitrogen atoms are doped into the graphene skeleton, including pyrrolic $\mathrm{N}$, pyridinic $\mathrm{N}$, and quaternary N (Figure S1a, Supporting Information). ${ }^{[28]}$ Pyrrolic N refers to $\mathrm{N}$ atoms bond into the five-membered ring that contribute two $\mathrm{p}$ electrons to the $\pi$ system. Pyridinic- $\mathrm{N}$ refers to nitrogen atoms at the edge of graphene planes, each of which is bonded to two carbon atoms and donates one p-electron to the aromatic $\pi$ system. Quaternary $\mathrm{N}$ refers to $\mathrm{N}$ atoms that substitute for $\mathrm{C}$ atoms in the hexagonal ring. ${ }^{[29]}$ For our sample, the deconvolution of the high resolution $\mathrm{N}$ 1s X-ray photoelectron spectrum (XPS, Figure S2b, Supporting Information) shows that the N1s peak can be resolved into three components centered at 398.1, 399.9, and $402.1 \mathrm{eV}$ representing pyridinic, pyrrolic, and quaternary type of $\mathrm{N}$ atoms doped in the graphene structure, respectively. ${ }^{[30]}$ Accordingly, the pyrrolic $\mathrm{N}$ dominates in the N-RGO prepared in this work.

We incorporated N-RGO into the perovskite film by dispersing $\mathrm{N}-\mathrm{RGO}$ in the $\mathrm{Pb}$-based perovskite precursor solution composed of mixed cations (formamidinium (FA) and methylammonium (MA)) and halides (I, Br), which was then spincoated on the thin mesoporous $\mathrm{TiO}_{2}$ layer. The solution had an excess of the lead compounds as recently reported by some of us. ${ }^{[14,31,32]}$ From the XRD patterns of the perovskite films, Figure S3 in the Supporting Information, it is evident that the materials fabricated from perovskite solutions in the absence and presence of N-RGO have a cubic perovskite phase with an almost unchanged crystal structure. ${ }^{[33]}$ Figure 1a,b show the SEM surface morphology images of perovskite films without and with N-RGO nanosheets. The perovskite/N-RGO exhibits almost full surface coverage with larger grains in comparison with the reference perovskite. The average grain size (Figure S4, Supporting Information) was estimated to be $\approx 196 \mathrm{~nm}$ for the pristine perovskite and $\approx 490 \mathrm{~nm}$ for the perovskite/NRGO. In addition, some grains were measured to be larger than $800 \mathrm{~nm}$ for the perovskite/N-RGO samples. As shown in Figure S4 in the Supporting Information, the grain size of the perovskite was almost unchanged by using RGO in the perovskite precursor solution. We hypothesize that the introduction of N-RGO supresses nucleation, leading to a decrease in the rate of growth compared to the control, similar to reports by others. ${ }^{[34]}$ This phenomenon is obvious from Figure 1c where the crystallization behaviour of the N-RGO films is twice as slow as the control. In turn, this enables the growth of only a few crystals, resulting in a film with limited number of large crystals.
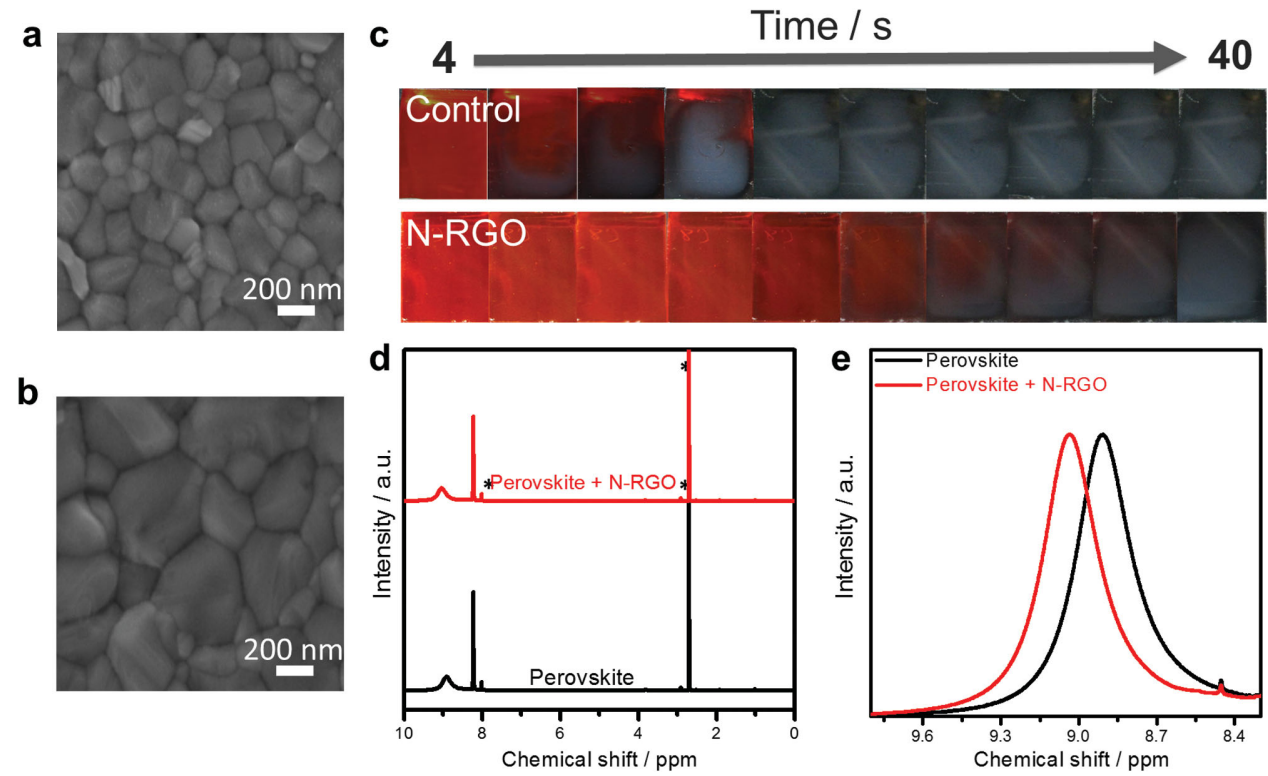

Figure 1. SEM top-view images of a) pristine perovskite and b) perovskite/N-RGO hybrid films. c) Photographs of perovskite films during crystallization at $100^{\circ} \mathrm{C}$. d) ${ }^{1} \mathrm{H}$ NMR of perovskite solution, and perovskite $+\mathrm{N}-\mathrm{RGO}$ collected in DMF- $\mathrm{d}_{7}$ (stars represent the peaks of DMF- $\mathrm{d}_{7}$ used as solvent). e) Magnification of the scale between 8.3 and $9.8 \mathrm{ppm}$. 
In order to gain insight into the mechanism of the increasing grain size of the perovskite in the presence of N-RGO, we collected liquid-state ${ }^{1} \mathrm{H}$ NMR spectra of the perovskite solution precursor in the absence and presence of N-RGO in deuterated $N, N$ dimethylformamide- $\mathrm{d}_{7}\left(\right.$ DMF- $\left._{7}\right)$ (Figure $\left.1 \mathrm{~d}\right)$. The peaks attributed to the solvent are marked in the figure. The broad peak at $\delta=8.91 \mathrm{ppm}$ belonging to the four hydrogen atoms attached to nitrogen atoms of the FA cation showed a downfield shift by adding N-RGO nanosheets to the solution. Figure $1 \mathrm{e}$ shows a magnification of the scale between 8.3 and $9.8 \mathrm{ppm}$. The observed downshift is due to the interaction between the hydrogen atoms of the FA and basic sites on N-RGO. As previously reported, ${ }^{[35]}$ protonation occurs at the most basic centers. Although some oxygen functional groups remain on N-RGO after hydrothermal treatment, they are acidic in nature. ${ }^{[36,37]}$ However, the nitrogen groups on graphene can be basic depending on their positions in the graphene structure. $\mathrm{Li}$ et al. ${ }^{[38]}$ evaluated and compared the basicity of the quaternary, pyridine, and pyrrole nitrogen groups on the graphene in terms of both Brønsted base and Lewis base by employing DFT calculations. The results of their work showed that the proton affinity of pyrrole and pyridine nitrogen groups, which is often invoked to characterize the Brønsted basicity, are significantly higher than pristine graphene. Additionally, in terms of Lewis basicity, the pyrrolic nitrogen group shows the strongest Lewis basicity. The XPS spectrum of N-RGO (Figure S2b, Supporting Information) shows that pyrrolic $\mathrm{N}$ is the main type among different nitrogen species in N-RGO prepared in this work. Therefore, ${ }^{1} \mathrm{H}$ NMR, accompanied by XPS results, confirms that N-RGO makes a different environment around FA hydrogen atoms slowing down the crystallization which leads to an increase in the perovskite grain size.

Larger grain size of perovskite/N-RGO enhances the lightharvesting capacity of the perovskite films and reduces the grain boundaries. ${ }^{[21]}$ As the UV-vis absorption spectra of the perovskite films with and without N-RGO (Figure S5, Supporting Information) show, presence of N-RGO in the perovskite precursor solution and its effect on the morphology of the perovskite film enhances the light absorption intensity which in turn enhances the light-harvesting capacity of the device.

We prepared mixed-PSCs by adding N-RGO to the precursor solution in order to understand its photovoltaic properties (Figure 2a). The perovskite films were formed in the absence and presence of N-RGO in the precursor solutions (see the Experimental Section). The devices were made of a stack of fluorine-doped tin oxide (FTO)/compact $\mathrm{TiO}_{2} /$ mesoporous$\mathrm{TiO}_{2} /$ mixed perovskite/Spiro-OMeTAD/gold as presented in Figure $2 \mathrm{~b}$. Figure $2 \mathrm{c}, \mathrm{d}$ shows the cross-sectional SEM images of the devices based on perovskite and perovskite/N-RGO hybrid capping layers. As seen in the figure, the perovskite capping layer is thicker by using the perovskite/N-RGO hybrid relative to the control device based on the perovskite alone. It shows that slowing down the crystallization of the perovskite in the presence of N-RGO induces an increase in the perovskite size in all dimensions, as we recently showed in our work that the larger grains resulted in thicker films. ${ }^{[16]}$

Figure 3a displays the current density-voltage $(J-V)$ curves of the reference and perovskite/N-RGO PSCs. We observed an increase in short circuit photocurrent density $\left(U_{\mathrm{SC}}\right)$ $\left(20.77-21.80 \mathrm{~mA} \mathrm{~cm}{ }^{-2}\right), V_{\mathrm{OC}}(1.12-1.15 \mathrm{~V})$, and slightly in FF (0.73-0.74) by using N-RGO in the perovskite precursor solution, which translated into an increase in the overall PCE from $17.3 \%$ to $18.7 \%$. It is important to note that the solar cells were measured at $10 \mathrm{mV} \mathrm{s}^{-1}$ scan rate and there is no large discrepancy between the reverse and forward device parameters (Figure S6, Supporting Information), indicating the reliability of the $J-V$ curve measurements. ${ }^{[39]}$ The detailed statistics of the photovoltaic parameters of perovskite/N-RGO and control devices are shown in Figure S7 in the Supporting Information. The detailed statistics clearly show that all the photovoltaic parameters improved when the perovskite films were grown in the presence of N-RGO. We ascribe the improvement in FF to the larger grains. The larger grains reduce the total grain boundaries to facilitate the transport and collecting of the charges through encountering less boundaries resulting in improved FF, which is in agreement with other studies. ${ }^{[10,11,16]}$

The largest improvement was seen for $J_{\mathrm{SC}}$ which is in agreement with the incident photon to current conversion efficiency shown in Figure S8 in the Supporting Information. We attribute the improvement in $J_{\mathrm{SC}}$ to the enhanced light-harvesting properties arising from the thicker perovskite film and from the reduction of small grains (Figure 1a,b) sitting atop the larger grains in the perovskite film. ${ }^{[16]}$ Better light-harvesting features of the perovskite/N-RGO are also confirmed by the enhanced absorption with respect to the pristine perovskite film (Figure S5, Supporting Information).

To shed light on the origin of the enhancement in FF, $J_{\mathrm{SC}}$, and $V_{\mathrm{OC}}$ with respect to the pristine PSC, we performed an additional control experiment by constructing perovskite/RGO hybrid based solar cells. By using RGO, we can exclude the
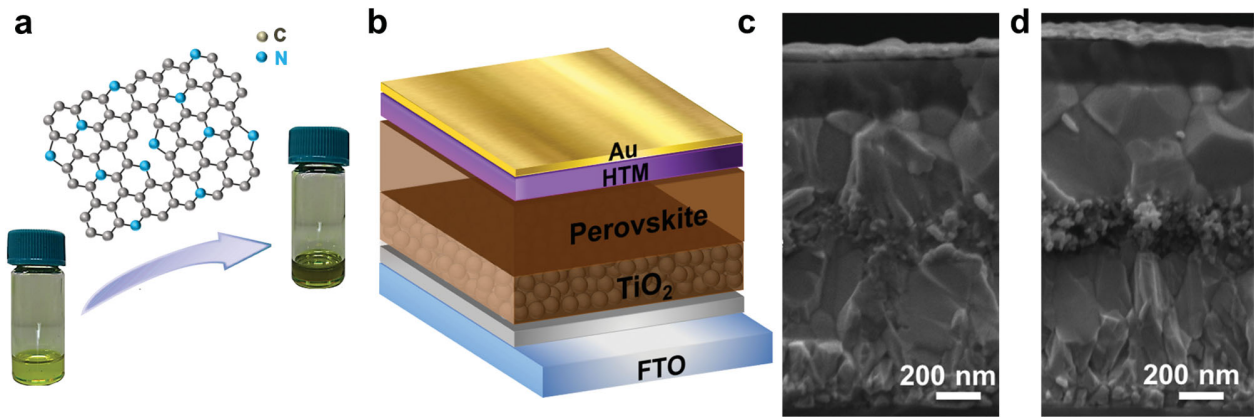

Figure 2. a) Pristine perovskite solution (left) and the perovskite/N-RGO hybrid solution (right). b) Schematic of full PSC. Cross-sectional SEM images of c) reference and d) perovskite/N-RGO hybrid solar cells. 
a

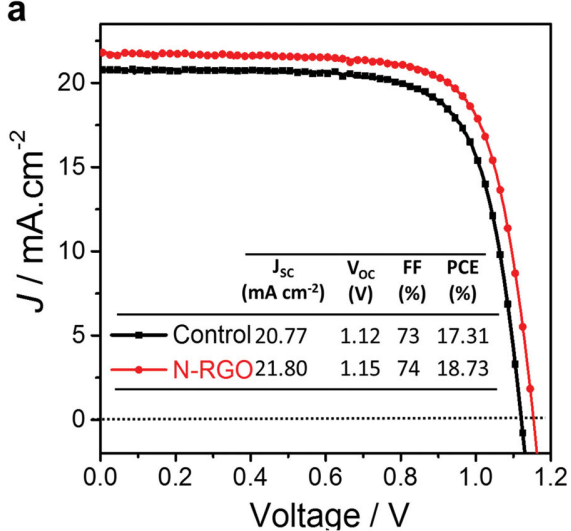

b

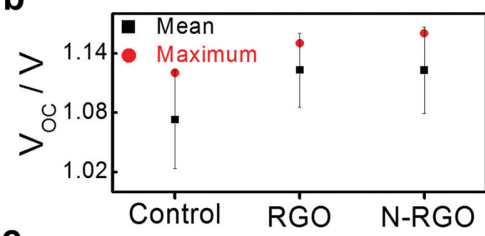

C

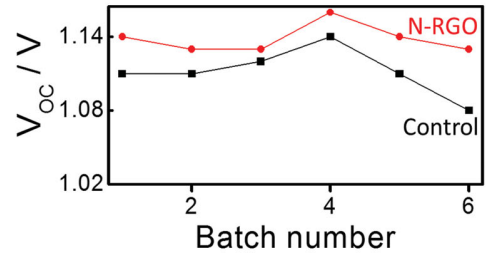

d

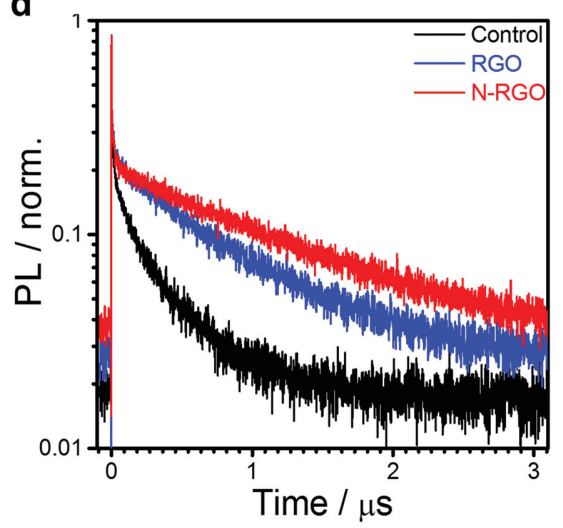

Figure 3. a) Current density-voltage characteristics for pristine perovskite (control) and perovskite/N-RGO hybrid solar cell (N-RGO). b) Average $V_{\mathrm{OC}}$ for the pristine perovskite (control), perovskite/RGO hybrid (RGO), and perovskite/N-RGO hybrid (N-RGO) solar cells. c) $V_{\text {OC }}$ of control, and devices with N-RGO layer on top of perovskite in six batches (Solid lines are a guide to the eye). The solar cells were measured at a scan rate of $10 \mathrm{mV} \mathrm{s}^{-1}$ and AM 1.5 G illumination with light intensity of $99 \mathrm{~mW} \mathrm{~cm}{ }^{-2}$. d) Time-resolved PL decay plots of pristine perovskite, perovskite/RGO, and perovskite/NRGO films. The measurements were performed from the perovskite film side.

effect of enlarging grains while the graphene sheets are present in the active layer. Figure $3 \mathrm{~b}$ shows the average values of $V_{\mathrm{OC}}$ of controls, perovskite/RGO, and perovskite/N-RGO in eight different batches. As it can be seen, the devices made by both perovskite/RGO and perovskite/N-RGO hybrids show a drastic enhancement in $V_{\mathrm{OC}}$. Figure $\mathrm{S} 9$ in the Supporting Information shows all the average device performance parameters in eight different batches. Perovskite/RGO based solar cells showed negligible change in the $J_{\mathrm{SC}}$ and FF. Other clear observation in Figure S9 in the Supporting Information is the improvement in the $J_{\mathrm{SC}}$ of the perovskite/N-RGO devices in comparison with those of the control and the perovskite/RGO devices. We can therefore infer that FF and $J_{\mathrm{SC}}$ are enhanced by the increased grain size and thicker perovskite film by using N-RGO in the perovskite solution. The enhanced performance of the devices with perovskite/RGO hybrid films is mainly attributed to the improved $V_{\mathrm{OC}}$. Therefore, we ascribe the enhancement of $V_{\mathrm{OC}}$ of perovskite/N-RGO solar cells to the presence of graphene sheets in the active layer rather than to the larger grain size. As seen in Figure S9 in the Supporting Information, the average PCE of devices increases in the sequence of pure perovskite, perovskite/RGO, and perovskite/N-RGO.

To further ensure that the improvement in $V_{\mathrm{OC}}$ is due to graphene sheets in the perovskite layer rather than the increase in perovskite grain size or thickness, we deposited N-RGO on top of the perovskite films after their crystallization to avoid the effect of N-RGO on crystallization (Figure S10a, Supporting Information). Although graphene sheets deposited on the perovskite film cannot entirely behave in the same way as when they are in the active layer, this experiment can provide insight into the role of graphene sheets in increasing the $V_{\mathrm{OC}}$. Figure $3 c$ shows the $V_{\mathrm{OC}}$ of the PSCs with and without N-RGO on top of the perovskite layer. It can clearly be seen that the $V_{\mathrm{OC}}$ is increased by N-RGO in all six batches without exception. Other photovoltaic parameters of the samples in six different batches are shown in Figure S10b in the Supporting Information. Unsurprisingly, the $J_{\mathrm{SC}}$ and FF remained unchanged, which further confirms our hypothesis that the graphene materials induce an increase in $V_{\mathrm{OC}}$. The improvement in $V_{\mathrm{OC}}$ was seen when we used N-RGO both in the perovskite layer and on top. This is consistent with surface passivation of the perovskite by graphene sheets improving the hole selectivity and retarding the electron-hole recombination.

In order evaluate the dynamics of charge carrier recombination of the perovskite film in the presence of graphene nanosheets, we utilized time-correlated single-photon counting measurements of control, perovskite/RGO, and perovskite/NRGO. Figure $3 \mathrm{~d}$ shows the photoluminescence (PL) decays for perovskite films and the fits are shown in Figure S11 in the Supporting Information. An alumina shell $(3 \mathrm{~nm}$ by atomic layer deposition) was used to avoid rapid quenching from the perovskite to the $\mathrm{TiO}_{2}$ particles, while simulating a device configuration. The observed decays were well-fitted using a biexponential function. The obtained lifetimes and amplitudes are listed in Table S1 in the Supporting Information. The PL decays are almost three times slower and over four times lower for perovskite films containing RGO and N-RGO, respectively, when compared to the control. This exemplifies the improved optoelectronic properties due to the superior quality of the perovskite/RGO and perovskite/N-RGO films. The longer lifetimes can be explained by the reduced non-radiative recombination due to the passivation of surface trap states by the graphene nanosheets. From these results, a higher $V_{\mathrm{OC}}$ for the devices prepared with perovskite/RGO and perovskite/N-RGO, is expected and indeed confirms our earlier conclusions on passivation at the perovskite/HTM interface.

In summary, we investigated the effect of the combination of two emerging materials, heteroatom-doped graphene and organic-inorganic lead halide perovskite in the active layer of the solar cells. By introducing N-RGO to the perovskite layer through dispersing it in the precursor solution, all the photovoltaic parameters were improved, which resulted in an improvement of the PCE from $17.3 \%$ to $18.7 \%$. The improved FF and $J_{\mathrm{SC}}$ are attributed mainly to the larger grains and thicker perovskite layer, which reduces the grain boundaries and improves light-harvesting properties. The ${ }^{1} \mathrm{H}$ NMR spectra showed that the basic nitrogen groups on N-RGO interact with FA cations in the blended perovskite solution resulting in enlarged grain 
size. On the other hand, the increase in $V_{\mathrm{OC}}$ is ascribed to surface-passivation of the perovskite by graphene sheets resulting in improved hole selectivity and reduced recombination at the perovskite/spiro interface. To the best of our knowledge, this is the first time that N-RGO has been used in PSCs to tune both morphological features and recombination dynamics. The introduction of this heteroatom-doped graphene as surfacepassivation and perovskite grain growth agent in the perovskite layer offers a new and effective tool towards the improvement of the PSCs.

\section{Experimental Section}

Synthesis of N-RGO and RGO Samples: The fabrication of graphite oxide as a raw material for fabrication of RGO and N-RGO was done via modified Hummers' method ${ }^{[37]}$ (Supporting Information). The $\mathrm{N}$-RGO sample was synthesized through a one-pot well-studied doping hydrothermal process with $\mathrm{GtO}$ as starting material and urea as the chemical doping agent. Typically, $160 \mathrm{mg}$ graphite oxide was dispersed in $80 \mathrm{~mL}$ deionized water using sonication for $1 \mathrm{~h}$. The colloidal graphene oxide suspension was centrifuged at $1000 \mathrm{rpm}$ for $5 \mathrm{~min}$ in order to remove any unexfoliated graphite oxide (GtO). Subsequently, $8 \mathrm{~g}$ urea was added to the suspension under sonication for $30 \mathrm{~min}$. The mixture was sealed in a $100 \mathrm{~mL}$ autoclave and maintained at $180^{\circ} \mathrm{C}$ for $12 \mathrm{~h}$. Then, the autoclave was naturally cooled to room temperature. The $\mathrm{N}$-RGO hydrogel was taken out with tweezers and dipped into deionized water to remove residual urea. It was washed with deionized water several times until the $\mathrm{pH}$ of the supernatant reached 7 . The product was dried at $80^{\circ} \mathrm{C}$ in a vacuum oven overnight. For comparison, RGO was also prepared under the same experimental parameters but without adding the urea into the graphene oxide aqueous dispersion.

Solar Cell Preparation: Electron selective layer preparation. The solar cells were fabricated on FTO substrates $\left(10 \Omega \mathrm{sq}^{-1}\right)$. The substrates were washed sequentially with detergent ( $2 \%$ Hellmanex in water), isopropanol, and acetone in an ultrasonic bath, and finally cleaned under UV-ozone treatment for $15 \mathrm{~min}$. A $\mathrm{TiO}_{2}$ blocking layer was deposited onto the substrates using spray pyrolysis of titanium diisopropoxide bis(acetylacetonate) precursor solution diluted in ethanol. The spraying was done at $450{ }^{\circ} \mathrm{C}$ followed by sintering process of the layers at $450{ }^{\circ} \mathrm{C}$ for $15 \mathrm{~min}$ before cooling down to room temperature. Subsequently, a mesoporous $\mathrm{TiO}_{2}$ layer was deposited on blocking-TiO $/$ FTO substrates via spin coating of $30 \mathrm{~nm}$ particle paste (Dyesol 30 NR-D) diluted in ethanol $\left(150 \mathrm{mg} \mathrm{mL}^{-1}\right)$ at $4000 \mathrm{rpm}$ for $20 \mathrm{~s}$. The layers were then dried at $100{ }^{\circ} \mathrm{C}$ for $10 \mathrm{~min}$ and sintered at $450^{\circ} \mathrm{C}$ for $30 \mathrm{~min}$ under dry air flow.

Perouskite Precursor Solution and Film Preparation: The precursor solution was prepared by dissolving an FAI (1 M), $\mathrm{MABr}(0.2 \mathrm{M}), \mathrm{Pbl}_{2}$ $(1.1 \mathrm{M})$, and $\mathrm{PbBr}_{2}(0.22 \mathrm{M})$ in anhydrous dimethylformamide:dimethyl sulfoxide (DMF:DMSO) 4:1 (v/v) to make $\mathrm{FA}_{0.85} \mathrm{MA}_{0.15} \mathrm{~Pb}\left(\mathrm{I}_{0.85} \mathrm{Br}_{0.15}\right)_{3}$ perovskite solution. The solution had excess of the lead compounds as recently shown by $\mathrm{Bi}$ et al. ${ }^{[14]}$ In order to make graphene incorporated perovskite precursor solution, $0.01 \mathrm{mg} \mathrm{mL}^{-1}$ of RGO and N-RGO were dispersed in the solvent (anhydrous DMF:DMSO 4:1 (v/v)) by $1 \mathrm{~h}$ sonication prior to making the perovskite solution. All other steps in device preparation were completely similar. The perovskite layers were deposited in a nitrogen-filled glovebox by spin coating the as-prepared perovskite precursor solution. The spin coating program included two consecutive steps, first at $1000 \mathrm{rpm}$ for $10 \mathrm{~s}$ and then at $6000 \mathrm{rpm}$ for $30 \mathrm{~s}$. During the second step, chlorobenzene was poured onto the spinning substrate $15 \mathrm{~s}$ before the end of the spin-coating program. The substrates were then annealed at $100{ }^{\circ} \mathrm{C}$ for $1 \mathrm{~h}$ on a hot plate in the nitrogen-filled glovebox to form crystalline perovskite.

Hole-Transporting Layer Preparation and Gold Evaporation: The HTM was subsequently deposited on the top of the perovskite layer by spin coating from the spiro-OMeTAD solution at $4000 \mathrm{rpm}$ for $20 \mathrm{~s}$. The spiro-OMeTAD solution, at a concentration of $70 \times 10^{-3} \mathrm{M}$ in chlorobenzene, was doped at a molar ratio of $0.5,0.03$, and 3.3 with bis(trifluoromethylsulfonyl)-imide lithium salt (Li-TFSI), $\operatorname{tris}(2-(1 \mathrm{H}-$ pyrazol-1-yl)-4-tert-butylpyridine (TBP))-cobalt(III) tris(bis(trifluoromethylsulfonyl)imide) (FK209), and TBP, respectively. Finally, $80 \mathrm{~nm}$ gold counter electrode was deposited by thermal evaporation under high vacuum.

\section{Supporting Information}

Supporting Information is available from the Wiley Online Library or from the author.

\section{Acknowledgements}

The authors thank Mr. Taisuke Matsui and Dr. Fabrizio Giordano for their help in providing the original recipe for the fabrication of the PSCs, and Dr. Wolfgang Tress for useful discussions. The authors would also like to thank Ferdowsi University of Mashhad for support of this project (Grant No. 3/29228). GRAPHENE project supported by the European Commission Seventh Framework Program under contract 604391 is gratefully acknowledged.

Received: May 25, 2016

Revised: July 14, 2016

Published online: August 12, 2016

[1] M. M. Lee, J. Teuscher, T. Miyasaka, T. N. Murakami, H. J. Snaith, Science 2012, 338, 643.

[2] H.-S. Kim, C.-R. Lee, J.-H. Im, K.-B. Lee, T. Moehl, A. Marchioro, S.-J. Moon, R. Humphry-Baker, J.-H. Yum, J. E. Moser, M. Grätzel, N.-G. Park, Sci. Rep. 2012, 2, 591.

[3] A. Kojima, K. Teshima, Y. Shirai, T. Miyasaka, J. Am. Chem. Soc. 2009, 131, 6050 .

[4] National Renewable Energy Laboratory (NREL), http://www.nrel. gov/ncpv/images/efficiency_chart.jpg (accessed: March 2013).

[5] Y. Rong, Z. Tang, Y. Zhao, X. Zhong, S. Venkatesan, H. Graham, M. Patton, Y. Jing, A. M. Guloy, Y. Yao, Nanoscale 2015, 7, 10595.

[6] T. C. Sum, N. Mathews, Energy Environ. Sci. 2014, 7, 2518.

[7] L. Etgar, P. Gao, Z. Xue, Q. Peng, A. K. Chandiran, B. Liu, M. K. Nazeeruddin, M. Grätzel, J. Am. Chem. Soc. 2012, 134, 17396.

[8] K. Wojciechowski, M. Saliba, T. Leijtens, A. Abate, H. J. Snaith, Energy Environ. Sci. 2014, 7, 1142.

[9] A. Yella, L.-P. Heiniger, P. Gao, M. K. Nazeeruddin, M. Grätzel, Nano Lett. 2014, 14, 2591.

[10] Z. Xiao, Q. Dong, C. Bi, Y. Shao, Y. Yuan, J. Huang, Adv. Mater. 2014, 26, 6503.

[11] W. Nie, H. Tsai, R. Asadpour, J.-C. Blancon, A. J. Neukirch, G. Gupta, J. J. Crochet, M. Chhowalla, S. Tretiak, M. A. Alam, H.-L. Wang, A. D. Mohite, Science 2015, 347, 522.

[12] Z. Liang, S. Zhang, X. Xu, N. Wang, J. Wang, X. Wang, Z. Bi, G. Xu N. Yuan, J. Ding, RSC Adv. 2015, 5, 60562.

[13] C. Roldan-Carmona, O. Malinkiewicz, R. Betancur, G. Longo, C. Momblona, F. Jaramillo, L. Camacho, H. J. Bolink, Energy Environ. Sci. 2014, 7, 2968.

[14] D. Bi, W. Tress, M. I. Dar, P. Gao, J. Luo, C. Renevier, K. Schenk, A. Abate, F. Giordano, J.-P. Correa Baena, J.-D. Decoppet, S. M. Zakeeruddin, M. K. Nazeeruddin, M. Grätzel, A. Hagfeldt, Sci. Adv. 2016, 2, e150117.

[15] H.-S. Kim, N.-G. Park, J. Phys. Chem. Lett. 2014, 5, 2927. 
[16] J. P. Correa-Baena, M. Anaya, G. Lozano, W. Tress, K. Domanski, M. Saliba, T. Matsui, T. J. Jacobsson, M. E. Calvo, A. Abate, M. Grätzel, H. Míguez, A. Hagfeldt, Adv. Mater. 2016, 28, 5031

[17] C. Bi, Y. Shao, Y. Yuan, Z. Xiao, C. Wang, Y. Gao, J. Huang, J. Mater. Chem. A 2014, 2, 18508

[18] N. K. Noel, A. Abate, S. D. Stranks, E. S. Parrott, V. M. Burlakov, A. Goriely, H. J. Snaith, ACS Nano 2014, 8, 9815.

[19] Y. Shao, Z. Xiao, C. Bi, Y. Yuan, J. Huang, Nat. Commun. 2014, 5, 5784.

[20] V. Somsongkul, F. Lang, A. R. Jeong, M. Rusu, M. Arunchaiya, T. Dittrich, Phys. Status Solidi RRL 2014, 8, 763.

[21] M. He, Y. Chen, H. Liu, J. Wang, X. Fang, Z. Liang, Chem. Commun. 2015, 51, 9659.

[22] K. S. Novoselov, V. I. Falko, L. Colombo, P. R. Gellert, M. G. Schwab, K. Kim, Nature 2012, 490, 192.

[23] K. Kim, H.-B.-R. Lee, R. W. Johnson, J. T. Tanskanen, N. Liu, M.-G. Kim, C. Pang, C. Ahn, S. F. Bent, Z. Bao, Nat. Commun. 2014, $5,4781$.

[24] G. S. Han, Y. H. Song, Y. U. Jin, J.-W. Lee, N.-G. Park, B. K. Kang, J.-K. Lee, I. S. Cho, D. H. Yoon, H. S. Jung, ACS Appl. Mater. Interfaces 2015, 7, 23521.

[25] J.-S. Yeo, R. Kang, S. Lee, Y.-J. Jeon, N. Myoung, C.-L. Lee, D.-Y. Kim, J.-M. Yun, Y.-H. Seo, S.-S. Kim, S.-I. Na, Nano Energy 2015, 12, 96.

[26] T. Sharifi, E. Gracia-Espino, H. Reza Barzegar, X. Jia, F. Nitze, G. Hu, P. Nordblad, C.-W. Tai, T. Wågberg, Nat. Commun. 2013, 4, 2319.
[27] E. K. Goharshadi, S. J. Mahdizadeh, J. Mol. Graphics Modell. 2015, 62, 74

[28] F. Zheng, Y. Yang, Q. Chen, Nat. Commun. 2014, 5, 5261.

[29] H. Wang, T. Maiyalagan, X. Wang, ACS Catal. 2012, 2, 781.

[30] L. Feng, Y. Chen, L. Chen, ACS Nano 2011, 5, 9611.

[31] F. Giordano, A. Abate, J. P. Correa Baena, M. Saliba, T. Matsui, S. H. Im, S. M. Zakeeruddin, M. K. Nazeeruddin, A. Hagfeldt, M. Graetzel, Nat. Commun. 2016, 7, 10379.

[32] J. P. Correa Baena, L. Steier, W. Tress, M. Saliba, S. Neutzner, T. Matsui, F. Giordano, T. J. Jacobsson, A. R. Srimath Kandada, S. M. Zakeeruddin, A. Petrozza, A. Abate, M. K. Nazeeruddin, M. Gratzel, A. Hagfeldt, Energy Environ. Sci. 2015, 8, 2928.

[33] T. Jesper Jacobsson, J.-P. Correa-Baena, M. Pazoki, M. Saliba, K. Schenk, M. Gratzel, A. Hagfeldt, Energy Environ. Sci. 2016, 9, 1706.

[34] F. Wang, H. Yu, H. Xu, N. Zhao, Adv. Funct. Mater. 2015, 25, 1120.

[35] A. Abate, D. J. Hollman, J. Teuscher, S. Pathak, R. Avolio, G. D'Errico, G. Vitiello, S. Fantacci, H. J. Snaith, J. Am. Chem. Soc. 2013, 135, 13538.

[36] A. M. Dimiev, L. B. Alemany, J. M. Tour, ACS Nano 2013, 7, 576.

[37] M. Hadadian, E. Goharshadi, A. Youssefi, J. Nanopart. Res. 2014, $16,1$.

[38] B. Li, X. Sun, D. Su, Phys. Chem. Chem. Phys. 2015, 17, 6691.

[39] J. A. Christians, J. S. Manser, P. V. Kamat, J. Phys. Chem. Lett. 2015, 6,852 . 